\title{
Itaconate ameliorates methicillin-resistant Staphylococcus aureus-induced acute lung injury through the Nrf2/ARE pathway
}

\author{
Gang Liu ${ }^{1,2 \#}$, Yaxian $\mathrm{Wu}^{2,3 \#}$, Sihao Jin ${ }^{1,2}$, Jiaojiao Sun ${ }^{1,2}$, Bin-Bin Wan ${ }^{2}$, Jiru Zhang ${ }^{4}$, Yingying Wang ${ }^{2}$, \\ Zhi-Qi Gao ${ }^{2}$, Dan Chen ${ }^{2}$, Shengpeng Li ${ }^{2}$, Qingfeng Pang ${ }^{2}$, Zhiqiang Wang ${ }^{1}$ \\ ${ }^{1}$ Department of Cardiothoracic Surgery, Affiliated Hospital of Jiangnan University, Wuxi, China; ${ }^{2}$ Wuxi School of Medicine, Jiangnan University, \\ Wuxi, China; ${ }^{3}$ Food Science and Technology, Jiangnan University, Wuxi, China; ${ }^{4}$ Department of Anesthesiology, Affiliated Hospital of Jiangnan \\ University, Wuxi, China \\ Contributions: (I) Conception and design: Z Wang, Q Pang; (II) Administrative support: D Chen; (III) Provision of study materials or patients: Q \\ Pang, Y Wu; (IV) Collection and assembly of data: G Liu, Y Wu, S Jin; (V) Data analysis and interpretation: ZQ Gao, D Chen, S Li, G Liu; (VI) \\ Manuscript writing: All authors; (VII) Final approval of manuscript: All authors. \\ \#These authors contributed equally to this work. \\ Correspondence to: Qingfeng Pang. Wuxi School of Medicine, Jiangnan University, 1800 Lihu Avenue, Wuxi 214122, China. \\ Email: qfpang@jiangnan.edu.cn; Zhiqiang Wang. Department of Cardiothoracic Surgery, Affiliated Hospital of Jiangnan University, No. 1000 \\ Hefeng Road, Wuxi 214122, China. Email: wxsywzq@163.com.
}

\begin{abstract}
Background: Methicillin-resistant Staphylococcus aureus (MRSA) are a critical predisposing factor of sepsis in the clinic. As a product of human energy metabolism and immune response, itaconate can effectively reduce inflammation in the body. This research employed 4-octyl itaconate (4-OI) to illustrate that itaconate exerted anti-inflammatory effects to protect the body from acute lung injury (ALI) induced by MRSA.

Methods: HE staining and immunohistochemistry are used to evaluate the MRSA-induced ALI in mice. WB and qPCR were used to verify the effect of 4-OI on inflammation and oxidative stress caused by MRSA. Molecular docking was used to verify the binding sites of 4-OI and Keap1.

Results: We demonstrated that 4-OI treatment increased the survival ratio, attenuated the pathological damage, inhibited neutrophil infiltration, and reduced lung bacterial burden in the mouse MRSA pneumonia model. 4-OI decreased the expression of inflammatory factors by stimulating the Nrf2 in vivo and in vitro. Furthermore, 4-OI exerted its effect by promoting nuclear transport of Nrf2 in vitro. The results of molecular docking indicated that 4-OI bound to the pocket of Keap1 and exerted a stable interaction. Both Nrf2 inhibitors (ML385) and $\mathrm{Nrf2}^{-/-}$mice abolished the protective effect of 4-OI on MRSA-induced inflammation both in vitro and in vivo.
\end{abstract}

Conclusions: 4-OI prevents lung damage caused by MRSA bacteremia via activating Nrf2/ARE pathway.

Keywords: Methicillin-resistant Staphylococcus aureus (MRSA); sepsis; itaconate; acute lung injury (ALI); NF-E2related factor 2

Submitted Mar 15, 2021. Accepted for publication Apr 22, 2021.

doi: $10.21037 /$ atm-21-1448

View this article at: http://dx.doi.org/10.21037/atm-21-1448

\section{Introduction}

Methicillin-resistant Staphylococcus aureus (MRSA), a critical pathogen causing hospital-acquired infections, is a major global health problem $(1,2)$. MRSA infection is a life-threatening disorder because of the bacteria's strong capacity for antibiotic resistance. Patients with MRSA sepsis often suffer from acute lung injury (ALI), hypoxemia, and respiratory distress $(3,4)$. Due to the continuous emergence of drug-resistant strains, MRSA-induced ALI is associated with high mortality and morbidity worldwide (5). Therefore, new drugs to treat patients with MRSA-induced ALI are urgently needed. 
Itaconate, a product of human energy metabolism and immune responses, has strong anti-inflammatory and antioxidant effects. Itaconate can also exert antibacterial effects by inhibiting isocitrate lyase (6). The antiinflammatory activity of itaconate has attracted increasing attention, with several studies reporting that itaconate dampens the inflammatory response in lipopolysaccharide (LPS)-treated macrophages and mice. Itaconate also inhibits the expression of cytokines and protects mice from ischemia-reperfusion injury (7). As derivatives of itaconate that can penetrate biological membranes, dimethyl itaconate (DI) and 4-octyl itaconate (4-OI) were found to decrease proinflammatory cytokine production in macrophages and protect mice against LPS-induced lethality (8). Additionally, DI has been shown to exert therapeutic effects against fungal infectious diseases, including imiquimodinduced psoriasis-like skin inflammation (9) and Aspergillus fumigatus-induced fungal keratitis in mice (10). However, the protective effect of itaconate against Gram-positive bacteria (e.g., MRSA)-induced ALI has not been studied.

Increasing evidence has indicated that nuclear factorerythroid 2 (NF-E2)-related factor $2(N r f 2)$ and its downstream gene, heme oxgenase-1 (HO-1) and $\mathrm{NAD}(\mathrm{P})$ $\mathrm{H}$ :quinone oxidoreductase 1 (NQO1), play important roles in the anti-inflammatory activity of many natural compounds. As a cell protective enzyme, HO-1 mainly catalyzes the catabolism of heme into ferrous iron, carbon monoxide and biliverdin. The degradation of the heme helps prevent its pro-oxidation effect. Biliverdin and its reduced bilirubin have effective ROS scavenging activity to resist oxidative stress. NQO1 can catalyze the reduction of quinone to hydroquinone, prevent quinone from forming semihydroquinone through a one-electron reduction reaction, and ultimately reduce the ROS generated by the oxidation of semihydroquinone. NQO1 can also prevent environmental stressors from causing oxidative damage to DNA and protect endogenous play an important role in antioxidants. For example, falcarindiol protects against LPS-induced acute intestinal inflammation through the Nrf2/HO-1 pathway (11). Angelica sinensis also activates $\mathrm{Nrf2/HO}-1$ and inhibits interleukin 1 beta (IL-1 $\beta$ ) and tumor necrosis factor alpha (TNF- $\alpha$ ) in LPS-induced RAW264.7 cells (12). Similarly, 4-OI promotes the nuclear localization of $\mathrm{Nrf} 2$ and thereby decreases IL- $1 \beta$ expression in LPS-treated macrophages and mice (9). However, it is unknown whether the anti-inflammatory effect of itaconate on MRSA-ALI is dependent on the Nrf2/ARE pathway.
This study was conducted to evaluate the molecular mechanisms by which 4-OI alleviates MRSA-ALI. The hypothesis that itaconate attenuates MRSA-ALI by activating the Nrf2/ARE pathway was tested using an MRSA-ALI mouse model, Nrf2-knockout $\left(\mathrm{Nrf2}^{--}\right)$mice, and Nrf2 inhibitors (ML385).

We present the following article in accordance with the ARRIVE reporting checklist (available at http://dx.doi. org/10.21037/atm-21-1448).

\section{Methods}

\section{Reagents and antibodies}

Lipoteichoic acid (LTA) from S. aureus (CAS\#56411-57-5) and dihydroethidium were obtained from Sigma-Aldrich (St. Louis, MO, USA). The following drugs were purchased from MedChemExpress (Monmouth Junction, NJ, USA) to treat cells or animals: ML385 (Cat\# HY-100523), 4-OI (Cat\# HY-112675), and corn oil (Cat\#HY-Y1888). Antibodies against GAPDH, Keap1, Nrf2, HO-1, and nitric oxide synthase (iNOS), and Cell Counting Kit-8 (CCK-8) were described in our previous report (13).

\section{Bacterial strains and growth conditions}

MRSA strain ATCC43300 was cultured and harvested as previously described (14). Briefly, this strain was grown to mid-log phase (OD $600 \mathrm{~nm}$ ) in Luria-Bertani (LB) with shaking at a rate of $200 \mathrm{rpm}$ in a constant temperature incubator at $37^{\circ} \mathrm{C}$. MRSA bacterial suspension was centrifuged, and the bacteria were resuspended in sterile phosphate-buffered saline (PBS). The bacterial suspension was diluted with sterile PBS and then plated onto LB agar to determine the bacterial concentration; the plated agar was stored in $50 \%$ glycerol at $-80{ }^{\circ} \mathrm{C}$.

\section{Animals}

C57BL/6 wild-type (WT) mice were obtained from Jiangnan University (Wuxi, Jiangsu, China). Male Nrf2knockout $\left(\mathrm{Nrf2}^{-/-}\right)$mice (8 weeks old, 18-25 g) were acquired from the Model Animal Research Center (Nanjing, China). Experiments were performed under a project license (No.: JN.No20191230c0400901) granted by ethics committee board of Jiangnan University, in compliance with China national or institutional guidelines for the care and use of animals. 


\section{MRSA-induced ALI mice model}

WT mice were grouped as follows (n=5): (I) intraperitoneal injection with saline solution (control), (II) intraperitoneal injection with $3 \times 10^{8}$ colony-forming unit/mouse MRSA bacteria (MRSA), and (III) intraperitoneal injection of MRSA and 4-OI (MRSA+4-OI). 4-OI was dissolved in the vehicle (dimethyl sulfoxide: corn oil =1:9). At $1 \mathrm{~h}$ after intraperitoneal injection of 4-OI $(25 \mathrm{mg} / \mathrm{kg})$ in mice, MRSA was intraperitoneally (i.p.) injected to construct an ALI model (13).

$\mathrm{Nrf2}^{-/-}$male mice were grouped as follows $(\mathrm{n}=5)$ : $\mathrm{Nrf2}^{-/-}$ control, $\mathrm{Nrf2}^{-/-} \mathrm{MRSA}$, and $\mathrm{Nrf2}^{-/-} \mathrm{MRSA}+4-\mathrm{OI}$. The 4-OI administration and MRSA bacteria were the same as those in WT mice. The mice in all groups were euthanized after 6 hours of treatment to obtain the experimental samples.

\section{Bronchoalveolar lavage fluid (BALF)}

The lung tissue of the mice was lavaged with sterile precooled PBS through the trachea. The BALF solution was centrifuged to obtain the supernatant. The protein concentration in the supernatant was determined using a bicinchoninic acid (BCA) kit (Beyotime, Shanghai, China). The total number of cells was determined by flow cytometry (BD C6, BD Biosciences, Franklin Lakes, NJ, USA).

\section{Acquisition and treatment of bone marrow-derived macrophages}

Bone marrow flushed from the femurs and tibias of WT C57BL/6 male mice were plated in Dulbecco's Modified Eagle's Medium (Hyclone, Logan, UT, USA) supplemented with $10 \%$ fetal bovine serum (Gibco, Grand Island, NY, USA), $1 \%$ penicillin-streptomycin (Gibco), and $10 \mathrm{ng} / \mathrm{mL}$ macrophage colony-stimulating factor (PeproTech, Rocky Hill, NJ, USA) for 6 days. Bone marrow-derived macrophages (BMDMs) were treated with 4-OI $(62.5 \mu \mathrm{M})$ or ML385 $(20 \mu \mathrm{M})$ for 4 hours and then challenged with LTA $(20 \mu \mathrm{g} / \mathrm{mL})$ for 4 hours.

\section{Content of myeloperoxidase (MPO) and malondialdebyde (MDA)}

Lung tissue was homogenized, and MPO and MDA were detected according to the manufacturer's instructions.

\section{Histopathology}

Lung tissues were fixed in $4 \%$ paraformaldehyde and embedded in paraffin. Hematoxylin and eosin (HE) staining was performed to observe lung tissue damage in the mice. The histological ALI scoring system, as described by Matute-Bello, was used (15).

\section{Immunobistochemistry}

Lung tissues were cut into 3 - $\mu \mathrm{m}$ sections from the embedded tissue, deparaffinized, and hydrated, and sodium citrate solution was used for antigen retrieval (Solarbio, Beijing, China). The sections were treated with specific serum proteins and immunostained with anti-MPO antibodies at a dilution of 1:100 (ab62141, Abcam, Cambridge, UK). After washing the sections with PBS, the sections were incubated with biotinylated immunoglobin $\mathrm{G}$ ( $\operatorname{IgG}$ ) at $37^{\circ} \mathrm{C}$ for 30 minutes. Finally, the sections were incubated in horseradish peroxidase-streptavidin for 30 minutes at room temperature, and the color reaction was developed using diaminobenzidine. The relative content of MPO in the lung tissue sections was determined by Image Pro Plus, as reported previously (13).

\section{Determination of the bacterial load in lung tissue}

To determine the bacterial load in the lung tissue, the lung tissues of mice were homogenized and diluted with sterile saline $\left(1: 10^{4}, 10^{5}, 10^{6}\right)$. Diluted lung tissue homogenate $(100 \mu \mathrm{L})$ was inoculated into LB agar plates and incubated overnight at $37^{\circ} \mathrm{C}$. The bacterial load in the lung tissue was determined by counting the number of bacteria on the LB agar plates at different concentrations (colony-forming unit/g).

\section{Survival rates}

To determine the effect of 4-OI on the survival time of MRSA-infected mice, WT and $\mathrm{Nrf2}^{-/-}$mice were divided into groups and treated as described above ( $\mathrm{n}=7-10$ /group) The survival time was recorded every day. Surviving mice in all groups were euthanized after 7 days.

\section{Cell culture and treatment}

RAW264.7 cells were purchased from the American Type 
Table 1 Primer sequences of qPCR

\begin{tabular}{lll}
\hline mRNA name & \multicolumn{1}{c}{ Sequence (5'-3') forward } & Sequence (5'-3') backward \\
\hline HO-1 & CAAGCCGAGAATGCTGAGTTCATG & GCAAGGGATGATTCCTGCCAG \\
Nrf2 & TTCAGCCAGCCCAGCACATC & CGTAGCCGAAGAAACCTCATTGTC \\
NQO-1 & TTCTGTGGCTTCCAGGTCTT & AGGCTGCTTGGAGCAAAATA \\
TNF- $\alpha$ & CATGAGCACAGAAAGCATGATCCG & AGCAGGAATGAGAAGAGGCTGAG \\
IL-1 $\beta$ & GCAACTGTTCCTGAACTCAACT & ATCTITTGGGTCCGTCAACT \\
IL-6 & ACAACCACGGCCTTCCCTACTT & CACGATTTCCCAGAGAACATGTG \\
GADPH & AGGTCGGTGTGAACGGATTTG & TGTAGACCATGTAGTTGAGGTCA \\
\hline
\end{tabular}

Culture Collection (Manassas, VA, USA) and treated with 4-OI $(62.5,125$, and $250 \mu \mathrm{M})$ or ML385 $(20 \mu \mathrm{M})$ for 4 hours, which was followed by challenge with LTA $(20 \mu \mathrm{g} / \mathrm{mL})$ for 4 hours. RAW264.7 cells were cultured in RPMI-1640 medium (Hyclone, Logan, UT, USA) supplemented with $10 \%$ fetal bovine serum (Gibco, Grand Island, NY, USA) and $1 \%$ penicillin streptomycin (Gibco). RAW264.7 cells were grown in an incubator containing $5 \% \mathrm{CO}_{2}$ at $37^{\circ} \mathrm{C}$.

\section{Determination of reactive oxygen species (ROS)}

RAW264.7 cells were seeded at a density of $1 \times 10^{6} \%$ well in 96-well plates. The cells were divided into the following groups: control, LTA, and LTA+4-OI (62.5 $\mu \mathrm{M})$. RAW264.7 cells were treated with 4 -OI $(62.5 \mu \mathrm{M})$ for 4 hours and then challenged with LTA $(20 \mu \mathrm{g} / \mathrm{mL})$ for 4 hours. Dihydroethidium $(10 \mu \mathrm{M})$ was added to a 96 -well plate containing RAW264.7 cells, which were incubated in the dark for 30 minutes. After washing the cells with PBS, fluorescence images were obtained using a Nikon TE-2000 microscope (Tokyo, Japan).

\section{Real-time quantitative polymerase chain reaction}

The lung tissue or cells were fully dispersed in TRIzol reagent (CWBIO, Beijing, China), and RNA was obtained according to the manufacturer's instructions. PrimeScript RT kit (Takara, Shia, Japan) and SYBR Mix (Yeasen, Shanghai, China) were used for the reverse transcriptase reaction and quantitative polymerase chain reaction (qPCR), respectively. The primer sequences are listed in Table 1.

\section{Western blot analysis}

The methods of extracting, separating, and transferring proteins from the lung tissues and cells have been previously described (13). Cytoplasmic and nuclear proteins were extracted using nuclear and cytoplasmic extraction reagents (Thermo Fisher Scientific, Waltham, MA, USA). The bands were visualized using an electrochemiluminescence system (Millipore, Billerica, MA, USA).

\section{Molecular modeling}

The three-dimensional structure of 4-OI was obtained from PubChem (https://pubchem.ncbi.nlm.nih. gov/\#query=3133-16-2). Keap1 (PDB ID: 1U6D) was downloaded from the RCSB protein database (https://www. rcsb.org/) and imported into AutoDock 4.2. Keap1 was cleaned and hydrogenated, and water was removed using Pymol software. Finally, a flexible program was selected for docking to evaluate the interaction sites and forces.

\section{Statistical analysis}

All data are expressed as the mean \pm SD. Differences between the 2 groups of data were compared by $t$-test. Cumulative survival rates were determined using the log-rank test. Differences were considered statistically significant a $\mathrm{P}$ value $<0.05$. GraphPad Prism 9.0 software (GraphPad, Inc., La Jolla, CA, USA) was used to draw the histograms and survival curves.

\section{Results}

\section{4-OI attenuated MRSA-induced ALI in mice}

HE staining results showed that the alveolar wall was deformed, and numerous inflammatory cells had infiltrated the alveolar space after MRSA infection. The lung injury 
score showed that 4-OI attenuated the aforementioned pathological changes in lung tissue caused by MRSA (Figure $1 A, B)$. MRSA infection caused inflammatory cells to migrate into the alveoli, whereas 4-OI reduced the infiltration of inflammatory cells into the lung tissue $(\mathrm{P}=0.0179$; Figure 1C). The protein content in the BALF, an indicator of lung vascular permeability, was significantly higher in the MRSA group than in the control group, whereas 4-OI decreased the total protein level in the BALF (Figure 1D). To further evaluate the effect of 4-OI on MRSA-induced lung neutrophil infiltration, we tested the activity and content of MPO in the lung tissue. The results showed that MPO activity was lower in the MRSA + 4-OI group than in the MRSA group $(\mathrm{P}=0.01429$; Figure $1 E)$. The results of MPO immunohistochemistry also showed that 4-OI treatment significantly reduced the infiltration of pulmonary neutrophils in the MRSA+4-OI group $(\mathrm{P}=0.0181$; Figure $1 F, G)$. Additionally, 4-OI treatment effectively reduced the number of bacteria in the lung organs $(\mathrm{P}=0.0452)$ (Figure 1H). Importantly, 4-OI treatment significantly increased the survival rate of mice(Figure 11).

\section{4-OI reduced inflammation and oxidative stress induced by MRSA in mice}

Real-time qPCR results showed that MRSA infection significantly increased the messenger RNA (mRNA) expression of $T N F-\alpha, I L-1 \beta$, and $I L-6$ in the lung tissue, while 4-OI treatment reduced the expression of these genes (Figure $2 A, B, C$ ). MDA content is an important indicator of the degree of tissue cell membrane peroxidation. We determined the concentration of MDA in the lung tissues and found that 4-OI significantly reduced the MDA content that had been increased by MRSA infection (Figure 2D). Notably, 4-OI upregulated the mRNA expression of Nrf2 and its downstream genes (NQO1 and HO-1) in MRSAinfected mice (Figure 2E,F,G).

\section{4-OI inbibited LTA-induced inflammatory response and oxidative stress injury in RAW264.7 cells}

The CCK- 8 assay results showed that 4-OI at concentrations of $62.5,125$, and $250 \mu \mathrm{M}$ did not affect the viability of RAW264.7 cells (Figure $3 A$ ). As iNOS is an important inflammatory mediator, we evaluated the effect of 4-OI on the iNOS content after LTA challenge in RAW264.7 cells. Western blotting showed that 4-OI (62.5, 125 , and $250 \mu \mathrm{M})$ significantly inhibited iNOS expression in RAW264.7 cells stimulated by LTA ( $\mathrm{P}=0.0244,0.0474$, 0.0434; Figure 3B,C). In RAW264.7 cells, LTA significantly increased the mRNA expression of TNF- $\alpha$, IL-1 $\beta$, and IL-6; various doses of 4-OI $(62.5,125$, and $250 \mu \mathrm{M})$ reduced the expression of these proinflammatory cytokines (Figure 3D,E,F). Dihydroethidium probes were used to detect the level of ROS in cells and results showed that 4-OI $(62.5 \mu \mathrm{M})$ decreased the cellular ROS content (Figure 3G). These results indicate that 4-OI reduced the expression of proinflammatory cytokines and oxidative stress damage induced by LTA in RAW264.7 cells.

\section{4-OI activated Nvf2/ARE patbway in LTA-stimulated macrophages}

Nrf2 mRNA level in RAW264.7 cells decreased after LTA stimulation, whereas 4-OI $(62.5,125$, and $250 \mu \mathrm{M})$ significantly increased Nrf2 expression. High expression of $N r f 2$ caused by 4-OI treatment increased the mRNA expression of downstream NQO1 and HO-1 (Figure 4A,B,C). Western blotting showed that 4-OI significantly increased the protein expression of $\mathrm{Nrf2}$ and $\mathrm{HO}-1$ in a dosedependent manner (Figure 4D,E,F).

Under oxidative stress conditions, the interaction between Keap1 and Nrf2 in the cytoplasm is disrupted, allowing $\mathrm{Nrf2}$ to dissociate into the nucleus. Therefore, we tested the expression of the Keap1 protein in the cells and found that 4-OI reduced Keap1 expression (Figure 4G). Furthermore, treatment with 4-OI increased the nuclear translocation of Nrf2 compared to that in the control and LTA stimulation groups (Figure 4H,I). Molecular docking analysis also showed that 4-OI inhibited the formation of hydrogen bonds with TYR-426 and ARG-442 in Keap1 (Figure 47,K).

\section{ML385 abolishes the anti-inflammatory effect of 4-OI in LTA-induced macrophages}

ML385 interacts directly with the Nrf2 protein by binding to the Neh1-binding region of Nrf2, preventing binding of the Nrf2-MAFG complex to the anti-oxidant response element (ARE) sequence and reducing transcriptional activity. ML385 abolished the effect of 4-OI on the promotion of the expression of $\mathrm{Nrf2}$ and $\mathrm{HO}-1$ (Figure $5 \mathrm{~A}, \mathrm{~B}, \mathrm{C}$ ). Additionally, ML385 upregulated the expression of proinflammatory cytokines $(T N F-\alpha, I L-1 \beta$, and $I L-6)$ in the ML385+4-OI group (Figure 5D,E,F). ML385 also abolished the effect of 4-OI on the promotion of the expression of Nrf2 and HO-1 and down-regulation of pro-inflammatory 
A

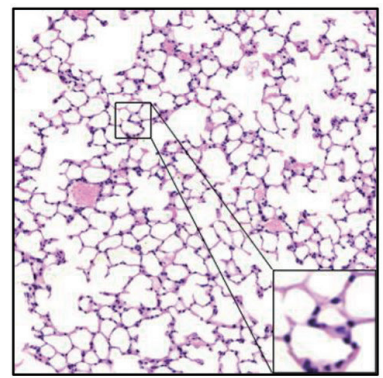

Control

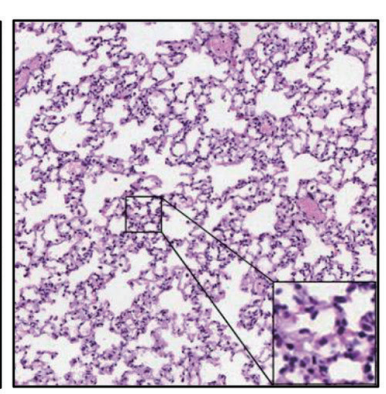

MRSA

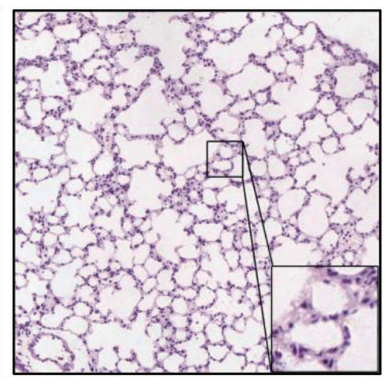

MRSA + 4-OI

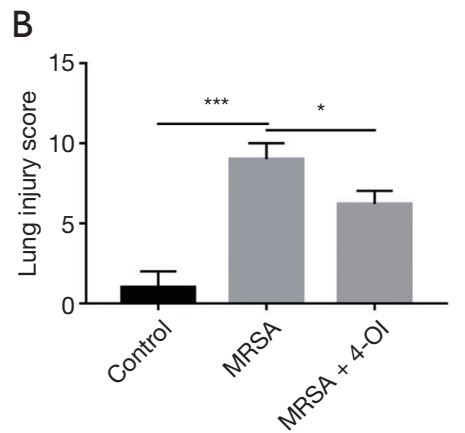

C
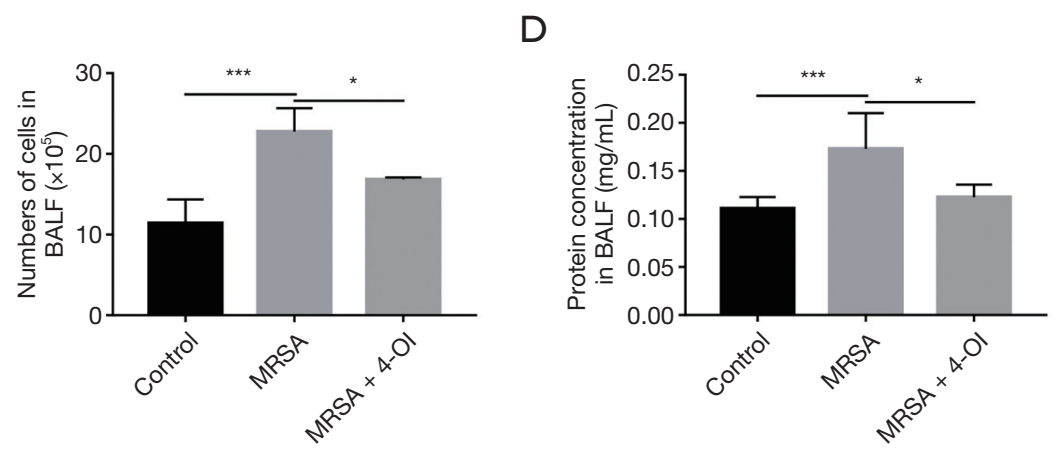

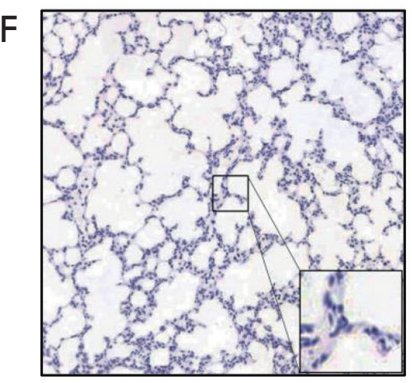

Control

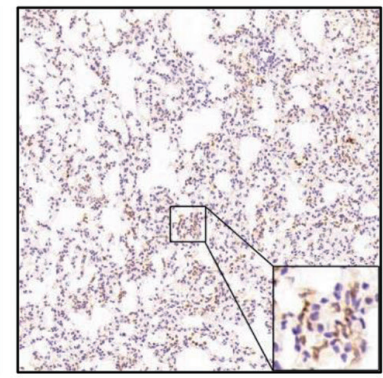

MRSA

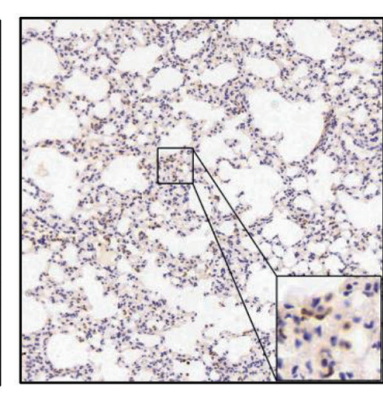

MRSA + 4-OI
E

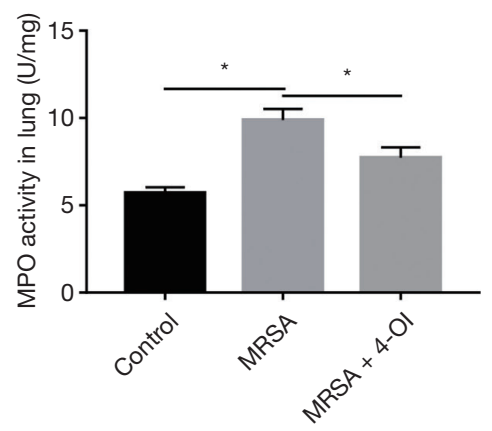

$\mathrm{H}$

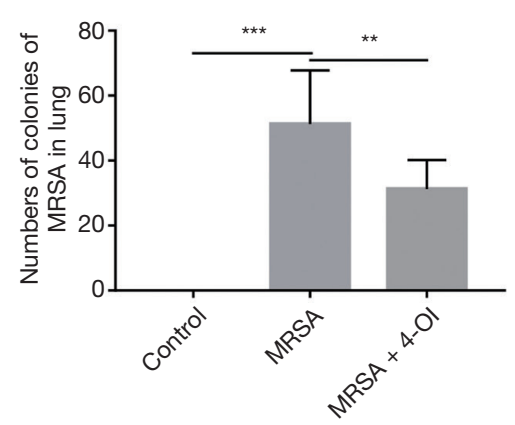

I

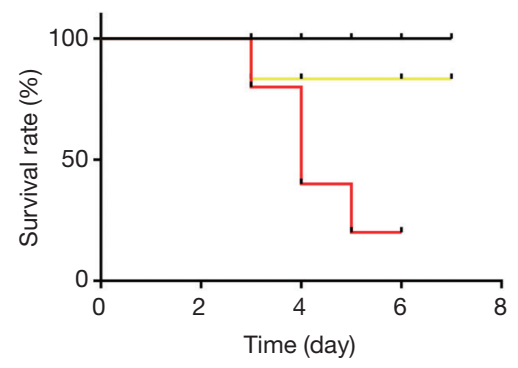

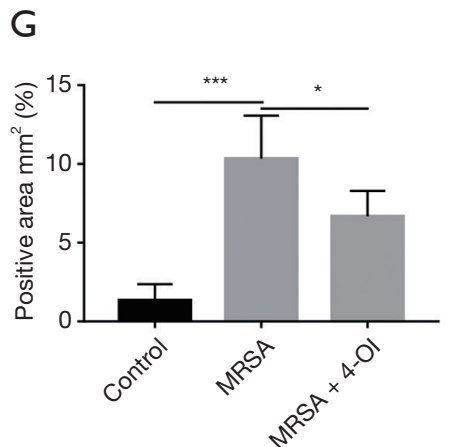

- Control

- MRSA

- MRSA + 4-OI

Figure 14 -OI alleviated MRSA-induced injury symptoms in the ALI mouse model. (A) HE staining (magnification, $\times 200$, bar =50 $\mu$; $n=5$ ). (B) Mouse lung injury score. (C) Total number of cells in BALF ( $n=5$ ). (D) Protein contents in BALF (n=5). (E) MPO activities in the lungs. (F) MPO immunohistochemistry (magnification, $\times 200$, bar $=50 \mu \mathrm{m} ; \mathrm{n}=5$ ). (G) Semiquantitative analysis of MPO immunohistochemistry. (H) MRSA bacterial burden in lung tissue. (I) Cumulative survival curves of MRSA-induced sepsis mice ( $\mathrm{n}=7-10$ /group). Data are represented as the mean \pm standard deviation $\left({ }^{*}, \mathrm{P}<0.05,{ }^{* *}, \mathrm{P}<0.01\right.$, and $\left.{ }^{* * *}, \mathrm{P}<0.001\right)$. MRSA, methicillin-resistant Staphylococcus aureus; ALI, acute lung injury; BALF, bronchoalveolar lavage fluid; MPO, myeloperoxidase. 
A
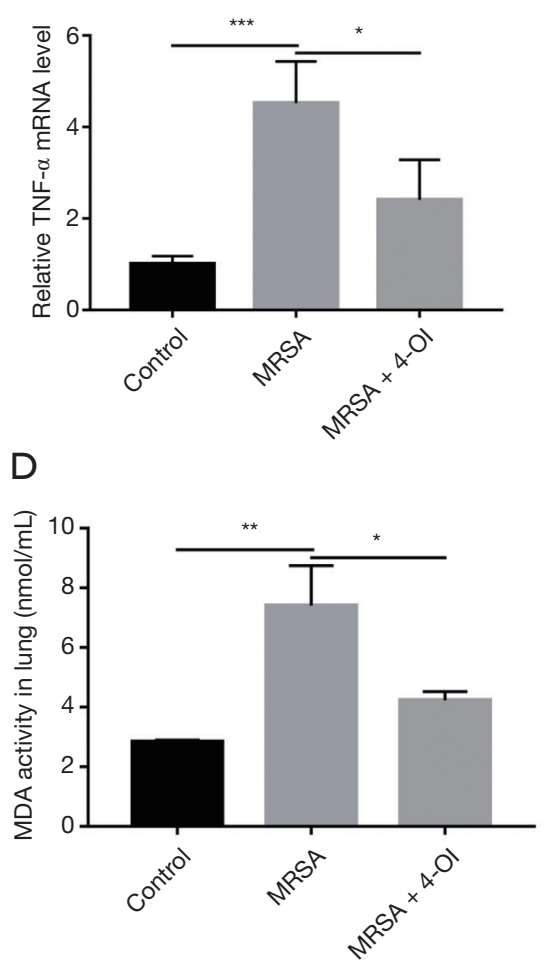

G

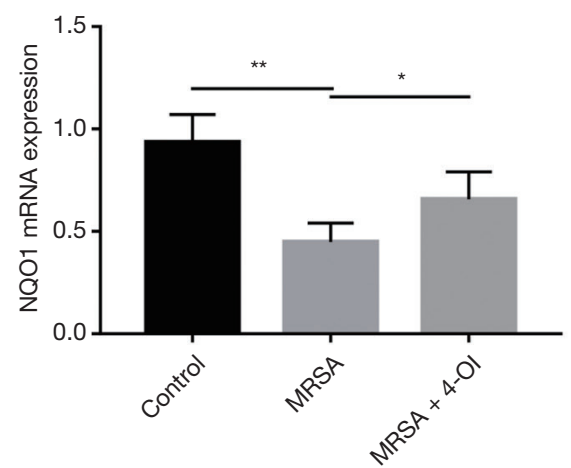

B

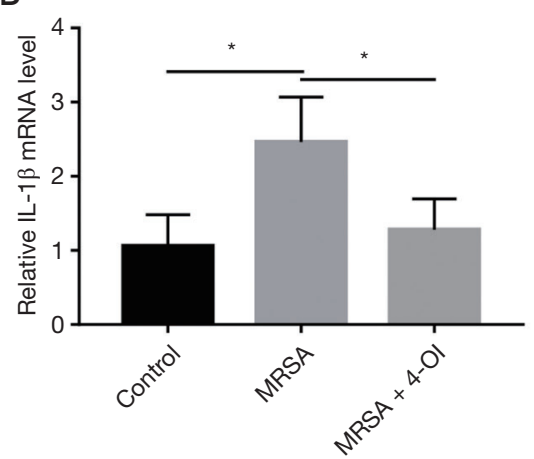

E

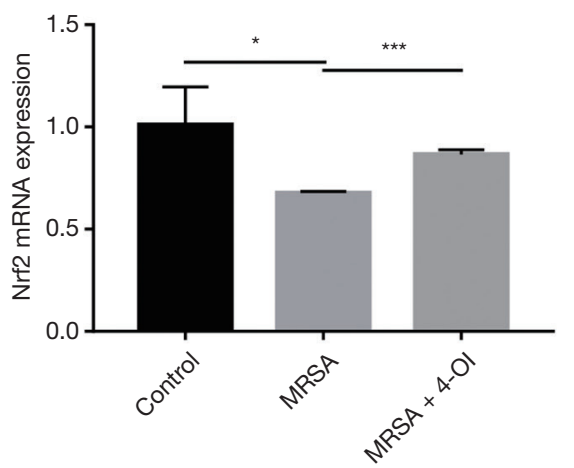

C

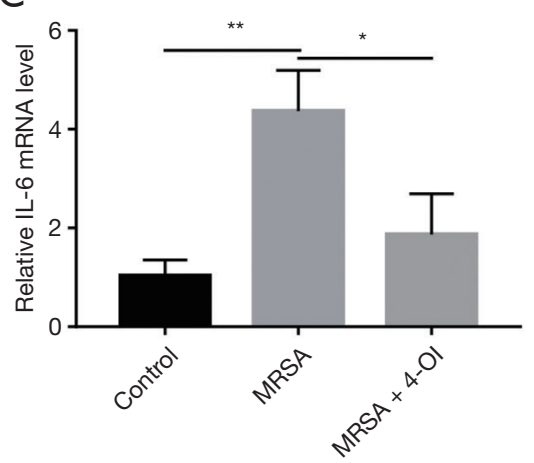

$\mathrm{F}$

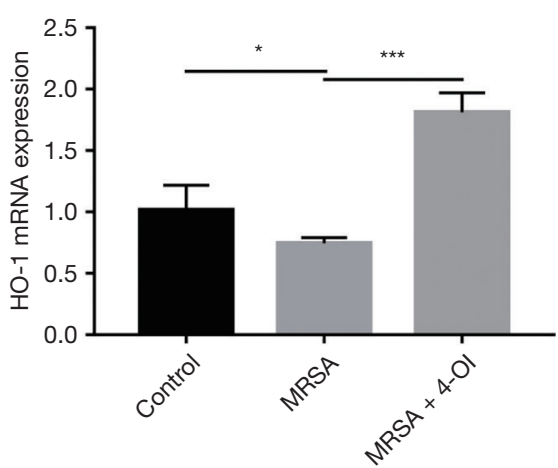

Figure $24-\mathrm{OI}$ reduced inflammation and oxidative stress induced by MRSA in vivo. (A) TNF- $\alpha$ mRNA levels. (B) mRNA levels of IL-1 $1 \beta$. (C) mRNA levels of IL-6. (D) MDA content in lung tissue. (E) mRNA levels of Nrf2. (F) mRNA levels of HO-1. (G) mRNA levels of NQO1. Data represent the mean \pm standard deviation. Mice in each group $=5$ ( ${ }^{*}, \mathrm{P}<0.05 ;{ }^{* *}, \mathrm{P}<0.01$; and $\left.{ }^{* * *}, \mathrm{P}<0.001\right) .4-\mathrm{OI}, 4$-octyl itaconate; MRSA, methicillin-resistant Staphylococcus aureus; TNF- $\alpha$, tumor necrosis factor alpha; MDA, malondialdehyde.

factors in BMDMs (Figure $5 G, H, I, \mathcal{Z}, K, L$ ). Taken together, these results show that Nrf2 contributes to the antiinflammatory effect of 4-OI in LTA-induced macrophages.

\section{Knockout of Nrf2 gene eliminated the protective effect of 4-OI on MRSA-induced ALI in mice}

To confirm the role of $\mathrm{Nrf} 2$ in the protective effect of
4-OI against MRSA-induced ALI in mice, we used $\mathrm{Nrf2}^{-/}$ mice for further validation. 4-OI treatment did not reverse the changes in the alveolar structure and infiltration of inflammatory cells in $\mathrm{Nrf2}^{-/-}$mice (Figure $6 A, B$ ). Additionally, 4-OI did not reduce the protein concentration (Figure $6 C$ ) or total cell number (Figure $6 D$ ) in the BALF or the MPO content of the lung tissue (Figure 6E,F,G). Furthermore, genetic ablation of Nrf2 increased the 
A

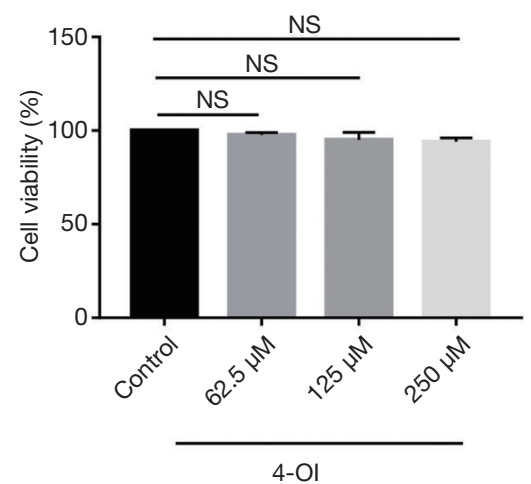

D

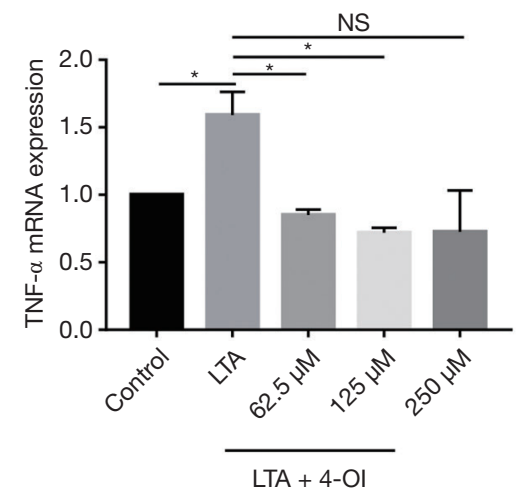

G

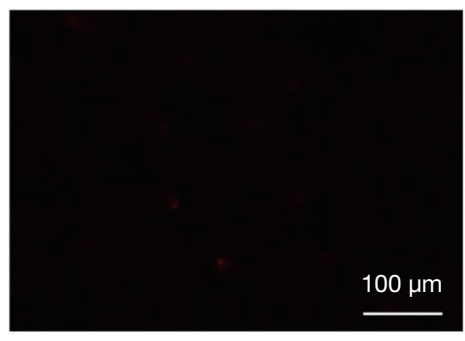

Control
B

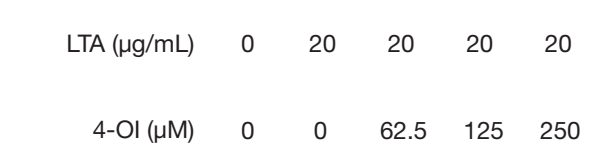

iNOS $(131 \mathrm{kDa})$

GAPDH $(36 \mathrm{kDa})$

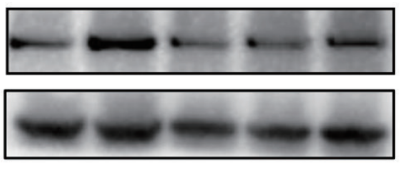

E
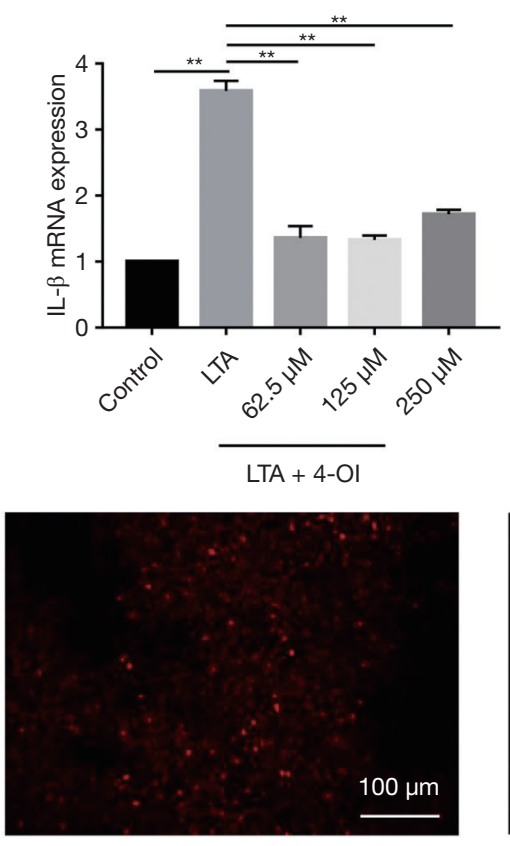

LTA
C

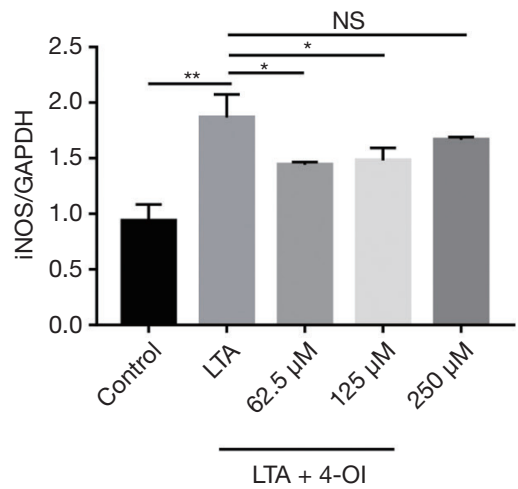

F

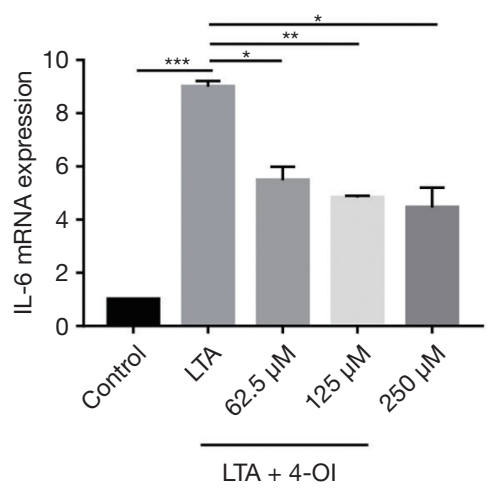

$\mathrm{LTA}+\mathrm{OI}(62.5 \mu \mathrm{mol} / \mathrm{mL})$

Figure 3 4-OI reduced inflammation and oxidative stress in LTA-induced RAW264.7 cells. (A) CCK-8 assay detected cell viability. (B,C) Western blotting of iNOS and statistical analysis. (D) TNF- $\alpha$ mRNA levels. (E) mRNA levels of IL-1 $\beta$. (F) mRNA levels of IL-6. (G) Dihydroethidium staining to detect ROS levels. Data represent the mean \pm standard deviation of 3 independent experiments $\left({ }^{*}, \mathrm{P}<0.05\right.$; **, $\mathrm{P}<0.01$; and $\left.{ }^{* * *}, \mathrm{P}<0.001\right)$. NS, no significant difference; 4-OI, 4-octyl itaconate; LTA, lipoteichoic acid; TNF- $\alpha$, tumor necrosis factor alpha.

bacterial load in the lungs, and 4-OI could not reverse this condition (Figure 6H). Importantly, 4-OI intervention did not improve the survival rate of MRSA-induced septic $\mathrm{Nrf}^{-/-}$mice (Figure 6I).

\section{Discussion}

In this study, we showed that 4-OI can alleviate MRSA- induced ALI by reducing the expression of proinflammatory cytokines and oxidative stress genes. The results of molecular docking and western blotting also showed that 4-OI decreased Keap1 levels and activated the Nrf2/ARE pathway to exert anti-inflammatory and antioxidant effects. Our study revealed that 4-OI is an effective therapeutic agent for MRSA-induced lung inflammation (Figure 7).

MRSA is the main pathogen causing acute pneumonia (16), 
A

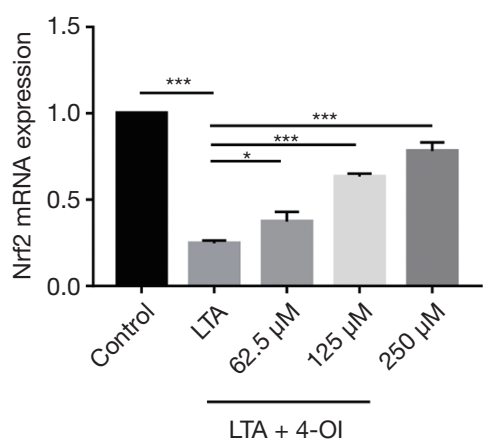

D

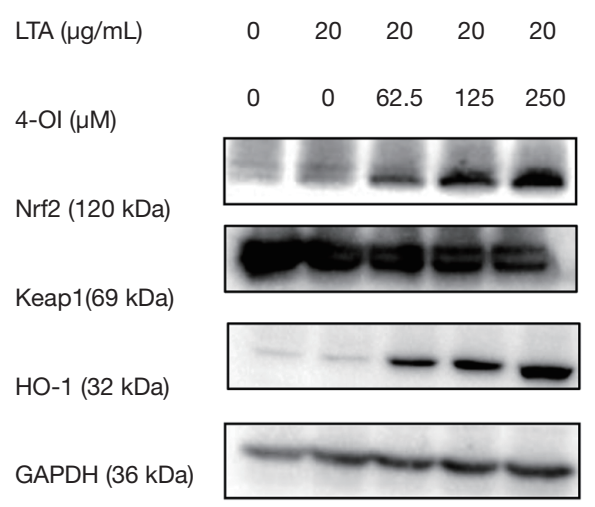

G

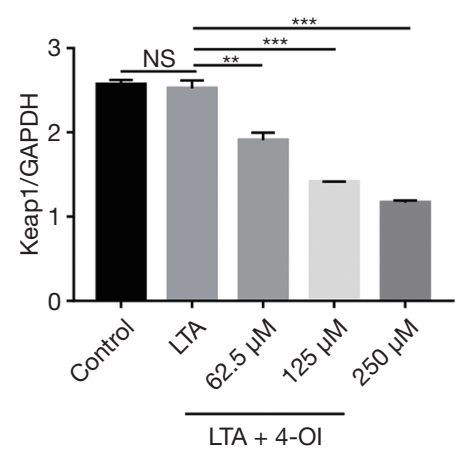

I

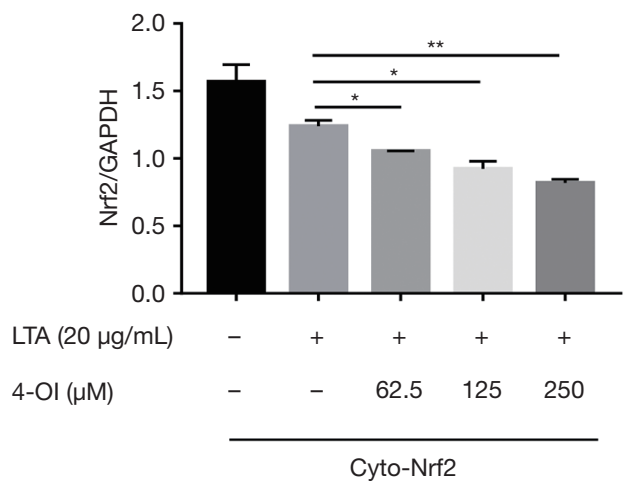

$E$
C
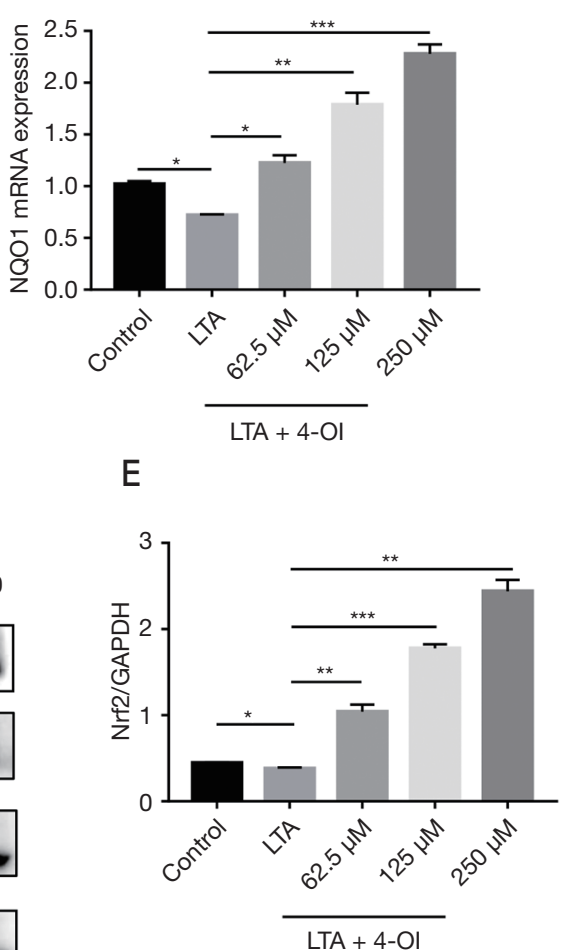

$\mathrm{H} \quad \mathrm{LTA}(\mu \mathrm{g} / \mathrm{mL})$

$4-\mathrm{OI}(\mu \mathrm{M})$

Cyto-Nrf2 (120 kDa)

GAPDH (36 kDa)

Nuc-Nrf2 (120 kDa)

Lamin B1 (68 kDa)

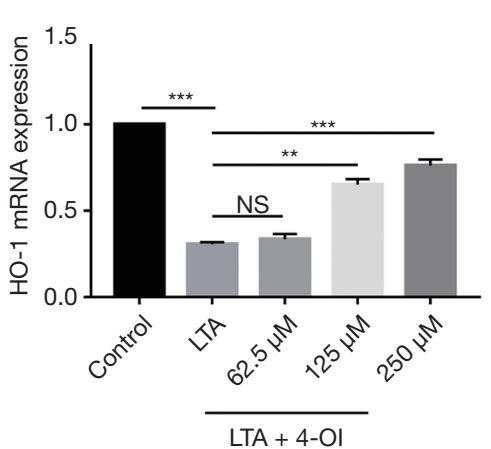

$\mathrm{F}$

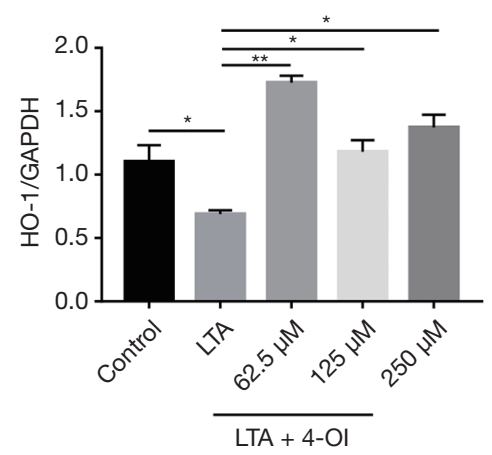

$\begin{array}{lllll}0 & 0 & 62.5 & 125 & 250\end{array}$
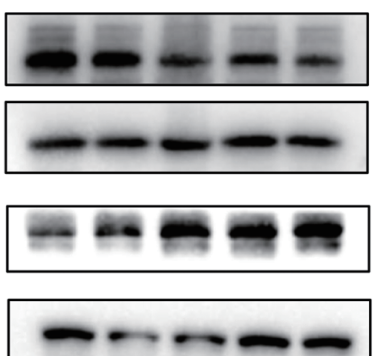

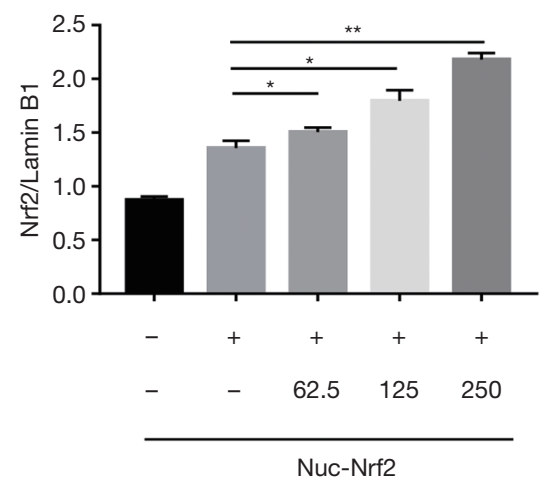



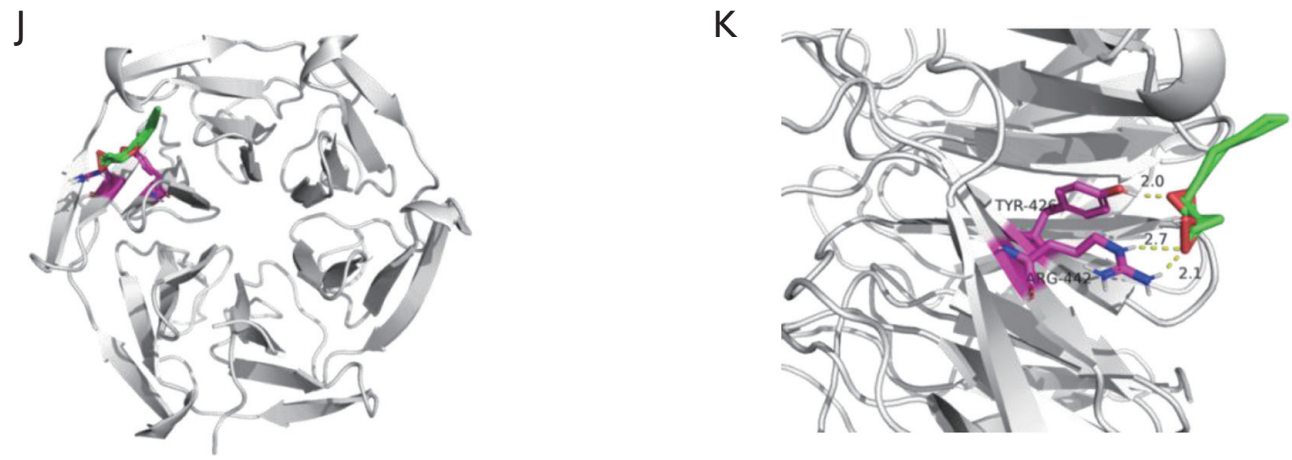

Figure 4 4-OI activated Nrf2 by reducing Keap1 and increasing Nrf2 protein content in LTA-induced macrophages. (A) mRNA levels of Nrf2. (B) mRNA levels of NQO1. (C) mRNA levels of HO-1. (D) Western blotting histograms for Nrf2, Keap1, and HO-1 proteins. (E,F,G) Statistical analysis of Nrf2, HO-1, and Keap1 levels in (D) histograms. (H) Western blot histograms of Nrf2 proteins in the cytoplasm and nucleus. (I) Statistical analysis of Nrf2 levels in (H) histograms. (J) Overall schematic of molecular docking. (K) Binding sites of 4-OI and Keap1. Data represent the mean \pm standard deviation of 3 independent experiments $\left({ }^{*}, \mathrm{P}<0.05 ;{ }^{* *}, \mathrm{P}<0.01\right.$; and $\left.{ }^{* * *}, \mathrm{P}<0.001\right)$. NS, no significant difference; 4-OI, 4-octyl itaconate; LTA, lipoteichoic acid.

chronic obstructive pulmonary disease, fibrosis (17) and other respiratory diseases in hospitals. The renal toxicity of vancomycin makes it difficult to effectively treat respiratory diseases caused by MRSA. As bacterial resistance continues to increase, the mortality rate of MRSA bacteremia has reached more than $20 \%(18)$.

Inflammation is an organism's defense response to irritants and pathogens (19). During the inflammatory response caused by Gram-positive bacteria, a large amount of inflammatory cell infiltration aggravates tissue damage by causing the release of inflammatory cytokines (20). Neutrophils are the first immune cells recruited to the injury site. Anti-inflammatory treatment is considered a promising method for treating the early stages of sepsis $(21,22)$. Previous studies have shown that itaconate, a product of human energy metabolism and the immune response, plays an indispensable role in inflammation. However, in the pathological process of MRSA-ALI, the continuous inflammatory response exceeds the regulatory ability of itaconate. However, 4-OI can enter cells where it is hydrolyzed into itaconate. Recent studies have demonstrated that 4-OI can exert anti-inflammatory effects and reduce oxidative stress effects in various cell types (23-25). As an important cell affected by $4-\mathrm{OI}$, macrophages play an important role in the process of oxidative stress. In this study, we also found that MRSA can cause a mortality rate of more than 50\%. 4-OI can effectively improve the survival rate of MRSA mice. After being stimulated by MRSA, both the content of MPO in lung tissue and the number of neutrophils localized by MPO increased significantly. 4-OI can significantly reduce the increase of MPO in MRSA mice. It has been reported that 4-OI reduces IL$1 \beta$ and IL-6 (not including TNF- $\alpha$ ) activity in LPStreated mouse macrophages and exerts anti-inflammatory effects (8). However, it should be noted that 4-OI reduces TNF- $\alpha$ expression in LTA-treated RAW264.7 and MRSAtreated mice. In addition, by activating Nrf2, 4-OI reduces the production of proinflammatory cytokines in human macrophages and peripheral blood mononuclear cells derived from patients with systemic lupus erythematosus (1). In conclusion, our results confirm that 4-OI treatment can reduce the mortality of MRSA-treated mice. 4-OI treatment can also reduce the inflammatory response and oxidative stress damage of MRSA-treated mice and LTAtreated macrophages.

The transcription factor Nrf2 plays a key role in regulating inflammation and oxidative stress (26-28). Activation of the Nrf2/ARE pathway increases the production of downstream enzymes, such as HO-1 and NQO1, thereby protecting the body from oxidative stress. In the resting state, Nrf2 binds to Keap1 in the cytoplasm, and Keap1 controls the expression of Nrf2 (26). When stimulated by the external environment, $\mathrm{Nrf2}$ can eliminate the binding of Keap1 and enter the nucleus to function as a transcription factor $(29,30)$. Itaconic acid esters increase the degradation of cysteine residues on Keap1, leading to further activation of $\mathrm{Nrf2}$ (26). A recent study found that 4-OI can alleviate $\mathrm{H}_{2} \mathrm{O}_{2}$-induced ROS generation and cell 
A

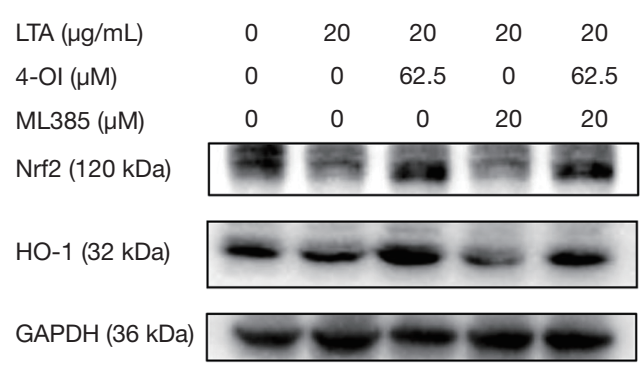

B

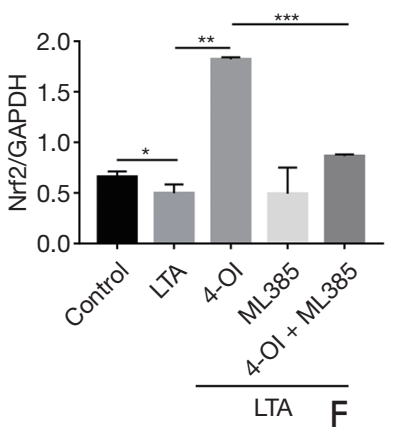

C

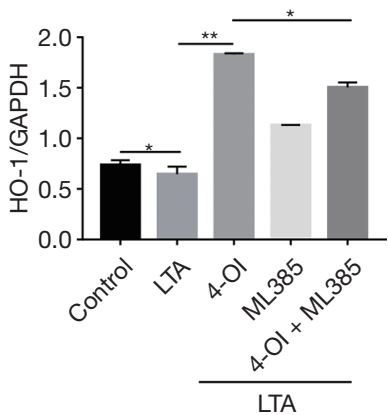

D

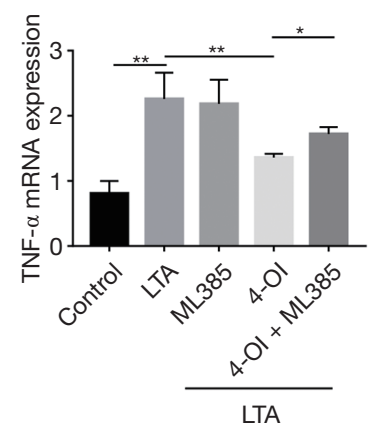

G

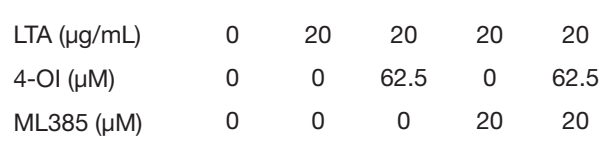

Nrf2 (120 kDa)

HO-1 (32 kDa)

GAPDH (36 kDa)

J

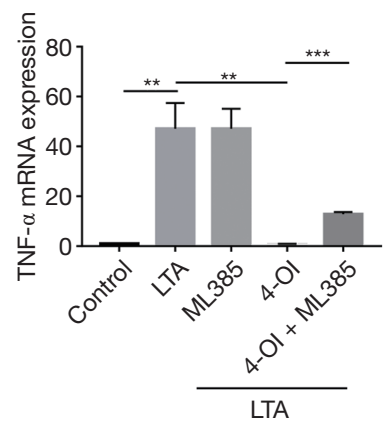

E

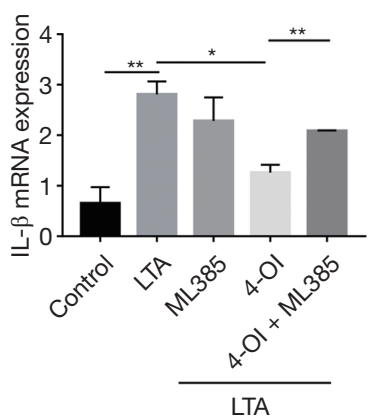

$\mathrm{H}$

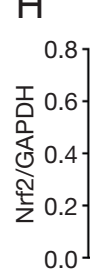

LTA

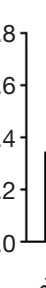

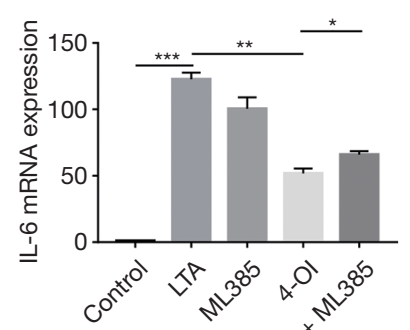
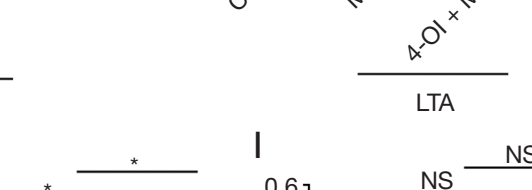

I
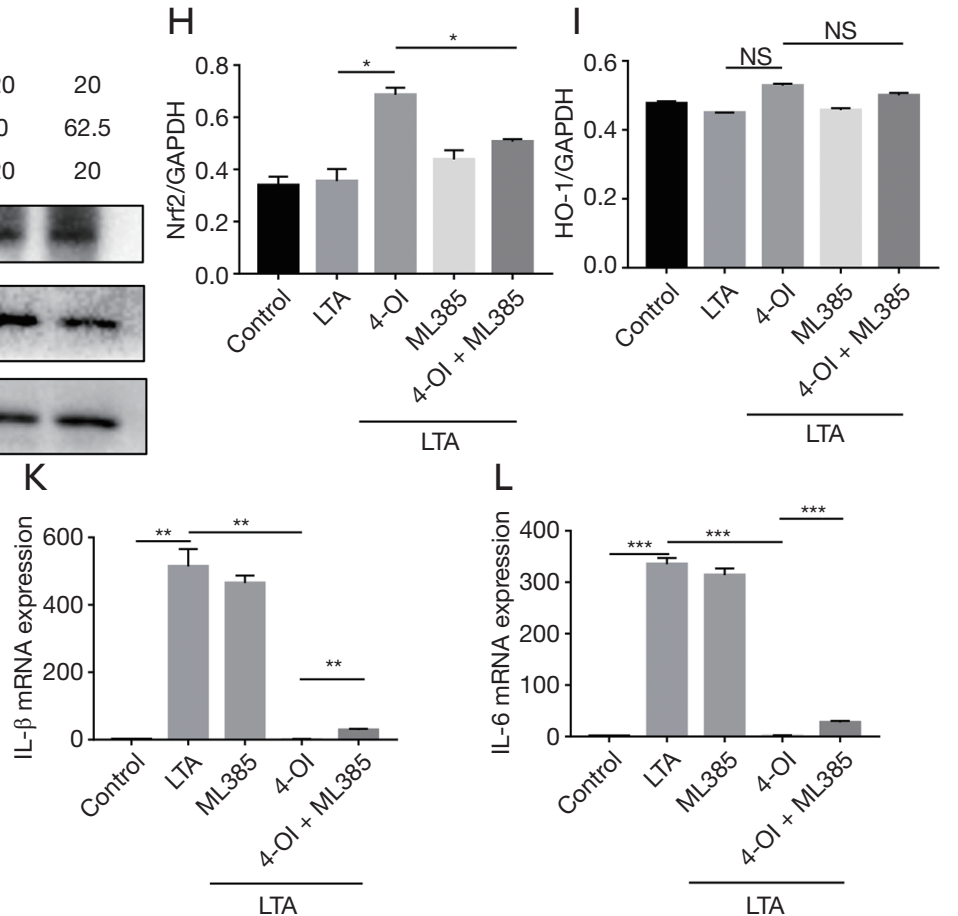

Figure $5 \mathrm{Nrf} 2$ inhibitors abolished the anti-inflammatory effect of 4-OI in LTA-induced macrophages. (A) Western blotting of Nrf2 and HO-1 levels in RAW264.7 cells. (B) Statistical analysis of Nrf2 level in (A) histograms. (C) Statistical analysis of HO-1 level in (A) histograms. (D,E,F) mRNA levels of TNF- $\alpha$, IL-11ß, and IL-6 were quantified by real-time qPCR in RAW264.7 cells. (G) Western blotting of Nrf2 and HO-1 levels in BMDMs. (H) Statistical analysis of Nrf2 level in (G) histograms. (I) Statistical analysis of HO-1 level in (G) histograms. (J,K,L) mRNA levels of TNF- $\alpha$, IL-11 \pm standard deviation of 3 independent experiments ( ${ }^{*}, \mathrm{P}<0.05$; ${ }^{* *}, \mathrm{P}<0.01$; and ${ }^{* * *}, \mathrm{P}<0.001$ ). NS, no significant difference; 4-OI, 4-octyl itaconate; LTA, lipoteichoic acid; BMDMs, bone marrow-derived macrophages. 
A

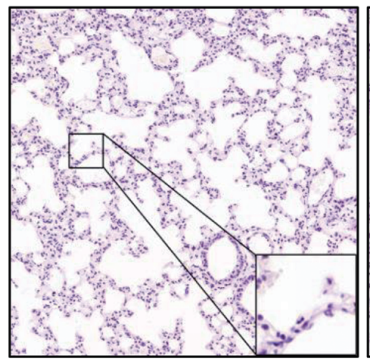

$\mathrm{Nrf2} \mathrm{KO}$

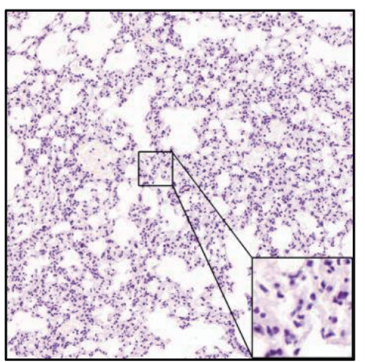

Nrf2 KO + MRSA

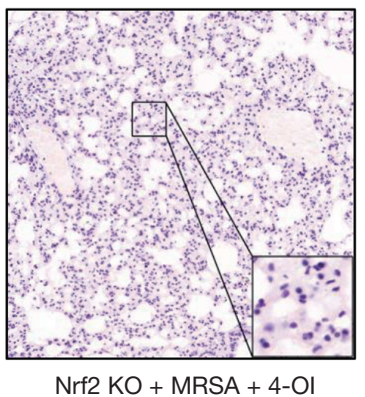

$\mathrm{Nrf2} \mathrm{KO}+\mathrm{MRSA}+4-\mathrm{OI}$

\section{B}

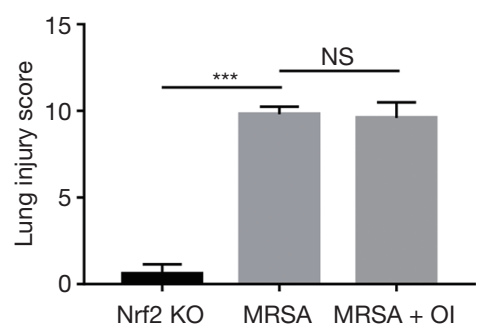

$E$

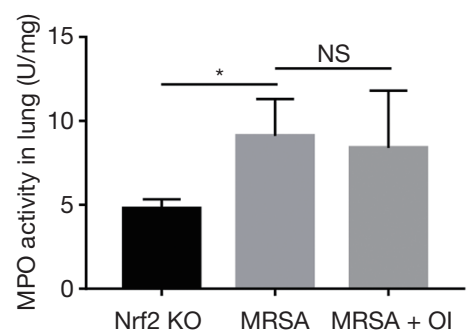

G

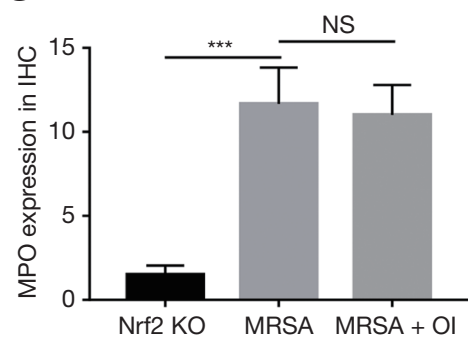

$\mathrm{F}$

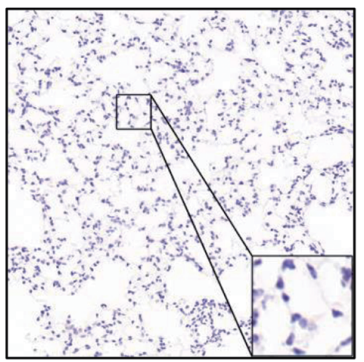

$\mathrm{Nrf} 2 \mathrm{KO}$
D

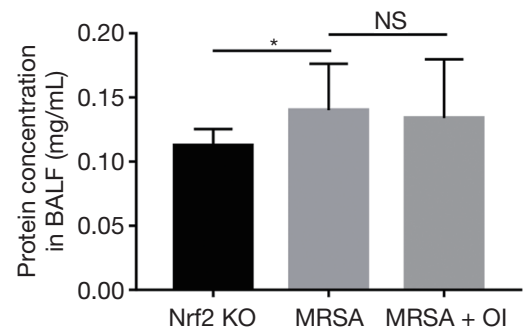

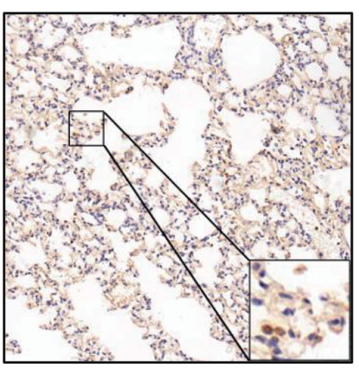

Nrf2 KO + MRSA

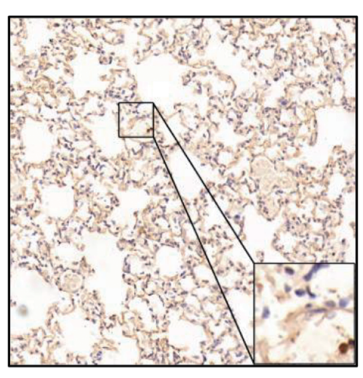

$\mathrm{Nrf2} \mathrm{KO}+\mathrm{MRSA}+4-\mathrm{OI}$

\section{I}
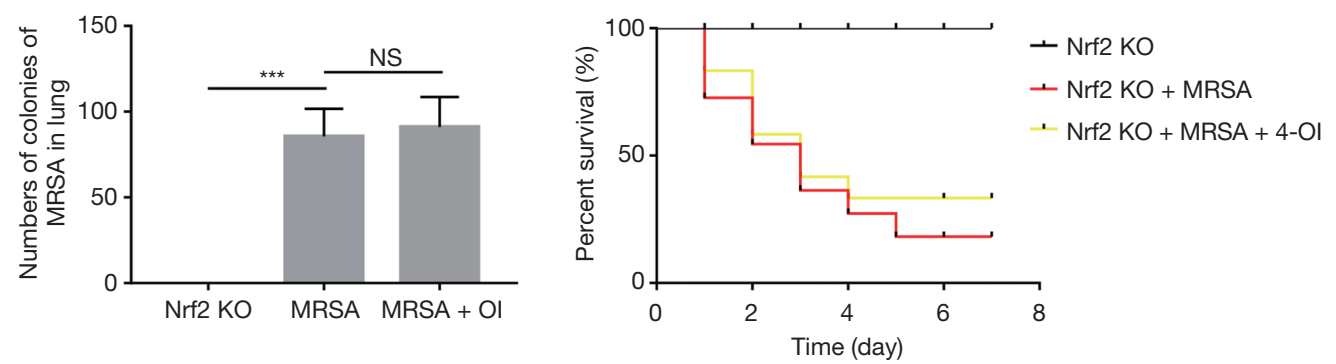

Figure 6 Knockout of Nrf2 gene eliminated the protective effect of 4-OI on MRSA-induced ALI. (A) HE staining (magnification, $\times 200$, bar $=50 \mu \mathrm{m} ; \mathrm{n}=5$ ). (B) Mouse lung injury score. (C) Total number of cells in BALF. (D) Protein contents in BALF (n=5). (E) Activity of MPO in lungs $(\mathrm{n}=3$ ). (F) MPO immunohistochemistry (magnification, $\times 200$, bar $=50 \mu \mathrm{m} ; \mathrm{n}=5$ ). (G) Semiquantitative analysis of MPO content by immunohistochemistry. (H) Determination of MRSA bacterial burden in lung tissue. (I) Effect of 4-OI on survival curves of MRSAinduced sepsis in $\mathrm{Nrf2}^{-/-}$mice ( $\mathrm{n}=7-10$ /group). Data represent the mean \pm standard deviation $\left.{ }^{*}, \mathrm{P}<0.05 ;{ }^{* * *}, \mathrm{P}<0.001\right)$. NS, no significant difference. MRSA, methicillin-resistant Staphylococcus aureus; 4-OI, 4-octyl itaconate; ALI, acute lung injury; MPO, myeloperoxidase; BALF, bronchoalveolar lavage fluid. 


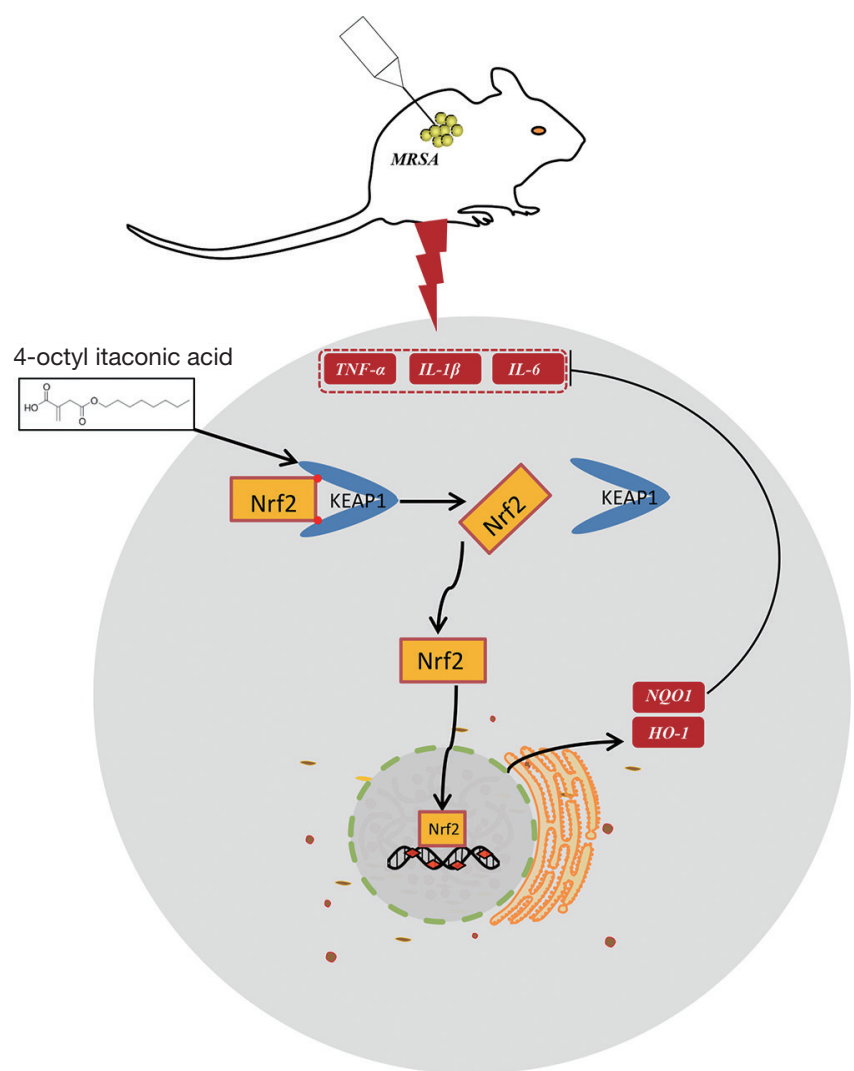

Figure 7 Mechanism of action of 4-octyl itaconate in alleviating MRSA-ALI. 4-OI promotes nuclear translocation of Nrf2 by binding to the amino acid residues of Keap1 (TYR-426 and ARG442); thereby activating the target genes downstream of Nrf2 (HO-1 and NQO1) to reduce MRSA-induced inflammation and oxidative stress. MRSA, methicillin-resistant Staphylococcus aureus; ALI, acute lung injury.

death in SH-SY5Y cells by disrupting the binding of Keap1NRF2 (31). Many studies have shown that 4-OI activates Nrf2/HO- 1 to reduce oxidative stress damage caused by various stimuli. This study is the first to predict the binding sites of 4-OI and Keap1 through molecular docking. 4-OI can activate the Nrf2/HO-1 pathway and promote the nuclear translocation of $\mathrm{Nrf} 2$ to resist inflammation and oxidation.

\section{Conclusions}

We proved that 4-OI promotes nuclear translocation of Nrf2 by binding to the amino acid residues of Keap1, thereby activating the Nrf2/ARE pathway to protect against MRSA-induced ALI. Thus, 4-OI is a potential therapeutic anti-inflammatory agent.

\section{Acknowledgments}

Funding: This study was supported by National Natural Science Foundation of China (No. 81871518 and 81901522), the China Postdoctoral Science Foundation (No. 2019M661730 and 2020M671347), the Natural Science Foundation of Jiangsu Province (No. BK20200602, BE2020634, and BK20191138), the Fundamental Research Funds for the Central Universities (No. JUSRP11955), the Top Talent Support Program for Young and Middle-aged People of Wuxi Health Committee (No. BJ2020049), and the Wuxi Commission of Health (No. T201824).

\section{Footnote}

Reporting Checklist: The authors have completed the ARRIVE reporting checklist. Available at http://dx.doi. org/10.21037/atm-21-1448

Data Sharing Statement: Available at http://dx.doi. org/10.21037/atm-21-1448

Conflicts of Interest: All authors have completed the ICMJE uniform disclosure form (available at http://dx.doi. org/10.21037/atm-21-1448). The authors report that this study was supported by National Natural Science Foundation of China (No. 81871518 and 81901522), the China Postdoctoral Science Foundation (No. 2019M661730 and 2020M671347), the Natural Science Foundation of Jiangsu Province (No. BK20200602, BE2020634, and BK20191138), the Fundamental Research Funds for the Central Universities (No. JUSRP11955), the Top Talent Support Program for Young and Middle-aged People of Wuxi Health Committee (No. BJ2020049), and the Wuxi Commission of Health (No. T201824). The authors have no other conflicts of interest to declare.

Ethical Statement: The authors are accountable for all aspects of the work in ensuring that questions related to the accuracy or integrity of any part of the work are appropriately investigated and resolved. Experiments were performed under a project license (No.: JN.No20191230c0400901) granted by ethics committee board of Jiangnan University, in compliance with China national or institutional guidelines for the care and use of animals. 
Open Access Statement: This is an Open Access article distributed in accordance with the Creative Commons Attribution-NonCommercial-NoDerivs 4.0 International License (CC BY-NC-ND 4.0), which permits the noncommercial replication and distribution of the article with the strict proviso that no changes or edits are made and the original work is properly cited (including links to both the formal publication through the relevant DOI and the license). See: https://creativecommons.org/licenses/by-nc-nd/4.0/.

\section{References}

1. Bambouskova M, Gorvel L, Lampropoulou V, et al. Electrophilic properties of itaconate and derivatives regulate the IkappaBzeta-ATF3 inflammatory axis. Nature 2018;556:501-4.

2. Channabasappa S, Chikkamadaiah R, Durgaiah M, et al. Efficacy of chimeric ectolysin P128 in drug-resistant Staphylococcus aureus bacteraemia in mice. J Antimicrob Chemother 2018;73:3398-404.

3. Cilloniz C, Dominedo C, Gabarrus A, et al. Methicillinsusceptible staphylococcus aureus in communityacquired pneumonia: Risk factors and outcomes. J Infect 2021;82:76-83.

4. De la Calle C, Morata L, Cobos-Trigueros N, et al. Staphylococcus aureus bacteremic pneumonia. Eur J Clin Microbiol Infect Dis 2016;35:497-502.

5. Gu L, Lin J, Wang Q, et al. Dimethyl itaconate protects against fungal keratitis by activating the $\mathrm{Nrf} 2 / \mathrm{HO}-1$ signaling pathway. Immunol Cell Biol 2020;98:229-41.

6. Hassoun A, Linden PK, Friedman B. Incidence, prevalence, and management of MRSA bacteremia across patient populations-a review of recent developments in MRSA management and treatment. Crit Care 2017;21:211.

7. Lakhundi S, Zhang K. Methicillin-Resistant Staphylococcus aureus: Molecular Characterization, Evolution, and Epidemiology. Clin Microbiol Rev 2018. doi: 10.1128/CMR.00020-18.

8. Lampropoulou V, Sergushichev A, Bambouskova M, et al. Itaconate Links Inhibition of Succinate Dehydrogenase with Macrophage Metabolic Remodeling and Regulation of Inflammation. Cell Metab 2016;24:158-66.

9. Mills EL, Ryan DG, Prag HA, et al. Itaconate is an antiinflammatory metabolite that activates $\mathrm{Nrf2}$ via alkylation of KEAP1. Nature 2018;556:113-7.

10. Nguyen TV, Alfaro AC, Young T, et al. Itaconic acid inhibits growth of a pathogenic marine Vibrio strain: A metabolomics approach. Sci Rep 2019;9:5937.
11. Saw CL, Wu Q, Su ZY, et al. Effects of natural phytochemicals in Angelica sinensis (Danggui) on Nrf2mediated gene expression of phase II drug metabolizing enzymes and anti-inflammation. Biopharm Drug Dispos 2013;34:303-11.

12. Stefanson AL, Bakovic M. Falcarinol Is a Potent Inducer of Heme Oxygenase-1 and Was More Effective than Sulforaphane in Attenuating Intestinal Inflammation at Diet-Achievable Doses. Oxid Med Cell Longev 2018;2018:3153527.

13. Zeng S, Chen D, Liu G, et al. Salvinorin A protects against methicillin resistant staphylococcus aureus-induced acute lung injury via Nrf2 pathway. Int Immunopharmacol 2021;90:107221.

14. Vincent JL, Sakr Y, Sprung CL, et al. Sepsis in European intensive care units: results of the SOAP study. Crit Care Med 2006;34:344-53.

15. Matute-Bello G, Downey G, Moore BB, et al. An official American Thoracic Society workshop report: features and measurements of experimental acute lung injury in animals. Am J Respir Cell Mol Biol 2011;44:725-38.

16. Kalil AC, Metersky ML, Klompas M, et al. Executive Summary: Management of Adults With Hospital-acquired and Ventilator-associated Pneumonia: 2016 Clinical Practice Guidelines by the Infectious Diseases Society of America and the American Thoracic Society. Clin Infect Dis 2016;63:575-82.

17. Goss CH, Muhlebach MS. Review: Staphylococcus aureus and MRSA in cystic fibrosis. J Cyst Fibros 2011;10:298-306.

18. Tong SYC, Lye DC, Yahav D, et al. Effect of Vancomycin or Daptomycin With vs Without an Antistaphylococcal beta-Lactam on Mortality, Bacteremia, Relapse, or Treatment Failure in Patients With MRSA Bacteremia: A Randomized Clinical Trial. JAMA 2020;323:527-37.

19. Yang J, Chen Y, Jiang K, et al. MicroRNA-106a Provides Negative Feedback Regulation in LipopolysaccharideInduced Inflammation by targeting TLR4. Int J Biol Sci 2019;15:2308-19.

20. Wheeler AP, Bernard GR. Acute lung injury and the acute respiratory distress syndrome: a clinical review. Lancet 2007;369:1553-64.

21. Tanaka T, Narazaki M, Kishimoto T. Immunotherapeutic implications of IL-6 blockade for cytokine storm. Immunotherapy 2016;8:959-70.

22. Venet F, Monneret G. Advances in the understanding and treatment of sepsis-induced immunosuppression. Nat Rev Nephrol 2018;14:121-37.

23. Sun X, Zhang B, Pan X, et al. Octyl itaconate inhibits 
osteoclastogenesis by suppressing Hrd1 and activating

Nrf2 signaling. FASEB J 2019;33:12929-40.

24. Song $\mathrm{H}, \mathrm{Xu} \mathrm{T}$, Feng X, et al. Itaconate prevents abdominal aortic aneurysm formation through inhibiting inflammation via activation of Nrf2. EBioMedicine 2020;57:102832.

25. Tian F, Wang Z, He J, et al. 4-Octyl itaconate protects against renal fibrosis via inhibiting TGF-beta/Smad pathway, autophagy and reducing generation of reactive oxygen species. Eur J Pharmacol 2020;873:172989.

26. Tocmo R, Parkin K. S-1-propenylmercaptocysteine protects murine hepatocytes against oxidative stress via persulfidation of Keap1 and activation of Nrf2. Free Radic Biol Med 2019;143:164-75.

27. Shao Y, Yu H, Yang Y, et al. A Solid Dispersion of Quercetin Shows Enhanced Nrf2 Activation and Protective Effects against Oxidative Injury in a Mouse Model of Dry Age-Related Macular Degeneration. Oxid Med Cell Longev 2019;2019:1479571.

Cite this article as: Liu G, Wu Y, Jin S, Sun J, Wan BB, Zhang J, Wang Y, Gao ZQ, Chen D, Li S, Pang Q, Wang Z. Itaconate ameliorates methicillin-resistant Staphylococcus aureusinduced acute lung injury through the Nrf2/ARE pathway. Ann Transl Med 2021;9(8):712. doi: 10.21037/atm-21-1448
28. Wang R, Luo Y, Lu Y, et al. Maggot Extracts Alleviate Inflammation and Oxidative Stress in Acute Experimental Colitis via the Activation of Nrf2. Oxid Med Cell Longev 2019;2019:4703253.

29. Cui G, Li L, Xu W, et al. Astaxanthin Protects Ochratoxin A-Induced Oxidative Stress and Apoptosis in the Heart via the Nrf2 Pathway. Oxid Med Cell Longev 2020;2020:7639109.

30. Han D, Gu X, Gao J, et al. Chlorogenic acid promotes the $\mathrm{Nrf} 2 / \mathrm{HO}-1$ anti-oxidative pathway by activating p21(Waf1/Cip1) to resist dexamethasone-induced apoptosis in osteoblastic cells. Free Radic Biol Med 2019;137:1-12.

31. Liu H, Feng Y, Xu M, et al. Four-octyl itaconate activates Keap1-Nrf2 signaling to protect neuronal cells from hydrogen peroxide. Cell Commun Signal 2018;16:81.

(English Language Editor: J. Gray) 